\title{
What is Needed Now is Policy Backup to Actively Integrate Fathers: Insights from Health Workers on Father Support for Exclusive Breastfeeding
}

\author{
Saratu O. Ajike* Motunrayo F. Olanrewaju \\ School of Public \& Allied Health, Babcock University, PO box 21244, Ilisan-Remo, Ogun State, Nigeria
}

\begin{abstract}
Health professionals are important in fostering a mother-infant-father triad towards breastfeeding success. Therefore, this study explored health workers' perspectives on father support for exclusive breastfeeding. A qualitative survey using semi-structured interviews was conducted among 11 health workers who were purposefully selected from Ikenne Local Government Area, southwest Nigeria. The participants had over 5 years' experience in maternal, newborn and child health services delivery. Content was analysed thematically. The major findings were (1) The health workers aknowledge their new role in the mechanism of enabling fathers to support breastfeeding, but also emphasized the need for health systems support. (2) Thinking outside of a patriarchal community, other forms of paternal social support were emphasized. (3) Community level strategies for active father support for breastfeeding were highlighted.The health workers have been making untailored efforts to involve men in health care. However, there is a need to develop appropriate policies to support the integration of father support programming into the current maternal and child health service delivery.
\end{abstract}

Keywords: Breastfeeding, Breastfeeding support, Father support, Health worker

DOI: $10.7176 / \mathrm{JHMN} / 71-10$

Publication date: February $29^{\text {th }} 2020$

\section{Introduction}

Breastfeeding has the potential to avert up to 823,000 deaths annually and potentially improve the socioeconomic status of children in the long term (Rollins et al., 2016). Moreover, it has the potential to reduce under-five mortality by $13 \%$ in developing countries (UNICEF, 2019) amongst other well-established benefits. Human milk also contains immunologic components and various biologically active substances that help infants utilize nutrients more efficiently and provides full protection against infections (WHO, 2019). Thus, it has both physiologic and nutritive benefits for the infant and benefits the woman and the society including those within her proximal and distal social environments (Johnson, Kirk, Rooks \& Muzik, 2016).

There has been worldwide consensus on the need to promote, support and protect breastfeeding as the first food for the newborn immediately following birth (WHO, 2019). Although breastfeeding is commonly practiced, rates still fall short of the $70 \%$ global target for the year 2030 to optimally protect women and children (WHO \& UNICEF, 2018). Early initiation of breastfeeding, maintenance of breastfeeding and Exclusive Breastfeeding (EBF) remain poorly practiced. Globally the early initiation rate is $42 \%$ with only $41 \%$ of infants aged 0 to 6 months who are breastfed exclusively (WHO \& UNICEF, 2018). In Eastern and South African EBF rates are at 55\%, while in West and Central Africa rates are lower at 34\% (UNICEF, 2019).

In Nigeria, a recent National Nutrition and Health Survey reported that up to $97.1 \%$ of children $0-23$ years are breastfed (NBS, 2018), while other breastfeeding practices are still suboptimal and far below the World Health Organization and United Nations Children's Fund (WHO/UNICEF) recommended values especially that of 6 months exclusive breastfeeding (NBS, 2018). Over $80 \%$ of infants do not receive human milk and colostrum within one hour of birth and only $27 \%$ of infants $0-5$ months are exclusively breastfed (NBS, 2018). Zonal EBF rates are between $21.3 \%$ and $43.9 \%$. Within the South West, these rates are as high as $55.3 \%$, however two states, Ondo and Ogun are still behind at $23.5 \%$ and $20.9 \%$ respectively (NBS \& UNICEF, 2018). As at the time of this study EBF rate of the study location was unavailable, however studies (Osibogun, Olufunlayo, \& Oyibo, 2018; Sholeye, Abosede \& Salako, 2015) that had been carried out in nearby towns had reported EBF rates of 39.2\% (Sholeye, Abosede \& Salako, 2015) and 28.5\% (Osibogun, Olufunlayo, \& Oyibo, 2018)

Factors that have been implicated in poor EBF practices in the South-west zone have been breast pain, difficult work schedule, perceived weight gain, poor feeding, and challenges with significant others (Agunbiade \& Opeyemi, 2012; Sholeye et al., 2015). Moreover, inadequate support from partners was also a dominant constraint to the practice of EBF (Agunbiade \& Opeyemi, 2012; Sholeye et al., 2015).

High quality support from both health professionals and fathers is essential for successful breastfeeding. Health professionals' support can positively influence the initiation, duration and women's experiences in breastfeeding (Battersby, 2014; Nieuwoudt \& Manderson, 2018). Similarly, fathers play critical roles in mothers' breastfeeding decisions and the maintenance of breastfeeding (Brown \& Davies, 2014). Thus, while health professionals remain an external strong influence, fathers are strong internal forces that can either work for or 
against optimal breastfeeding.

Studies (Brown \& Davies, 2014; Sheriff, Panton \& Hall, 2014) have revealed the need for focused interventions targeted at fathers to enable the provision of optimal support towards breastfeeding. Health professionals are in the position to offer these targeted services and motivate fathers to support breastfeeding Sheriff et al., (2014) noted that positive attitude towards breastfeeding can be influenced by the acknowledgement of the roles and contribution of fathers within the breastfeeding support framework in addition to attitudes of influential others and cultural norms (Rosin and Zakarija-Grković, 2016).

Studies (OlaOlorun \& Lawoyin, 2006; B. Utoo, Ochejele, Obulu \& P.M. Utoo, 2012) in Nigeria have investigated health workers' tangible support for breastfeeding and health worker's breastfeeding knowledge and attitudes towards breastfeeding in general. However, no attention has been paid to paternal support for breastfeeding despite increasing interest in seeking other innovative means of promoting improved EBF rates and more recent attention to the relevance of community support mechanisms of social support in enabling mothers breastfeeding optimally (WHO \& UNICEF, 2018). Moreover, it has been noted that perspectives of health care workers (HCWs) working with breastfeeding women can be an important element in breastfeeding support interventions (Flood, 2017).

Therefore, the study aimed to explore health workers' perspectives about father support for exclusive breastfeeding in a South-western suburban community in Nigeria. This paper is drawn from an exploratory phase of a larger study which is utilizing mixed methods to generate data that will be useful for developing fatherinvolved interventions in the promotion of exclusive breastfeeding in Nigeria and beyond.

\section{Materials and Methods}

\subsection{Design}

Using a qualitative research methodology underpinned by an interpretive paradigm this study conducted qualitative interviews among nurses and community health extension workers offering maternal and newborn health services. The intent of the researcher was to gain an understanding of father breastfeeding support in the local community and if health practitioners would consider a father-inclusive breastfeeding initiative as part of ongoing breastfeeding and exclusive breastfeeding promotion and also gain insights for a pilot community-based participatory breastfeeding intervention focused on expectant fathers. These objectives fit with the interpretive paradigm which allows subjectivity in the understanding a social phenomenon and gaining a full picture of the social issue (Bhattacherjee, 2012).

2.1.1 Sample/Participants

A sample of registered nurses and community health extension workers were recruited. They were purposefully selected based on length of experience in maternal and newborn service delivery. The inclusion criteria were, that they had 5 years or more of service experience and would voluntarily agree to participate. The exclusion criteria were that they had less than 5 years of experience and declined participation. All participants were recruited through an administrative head at the local government. 11 health workers took part in the study. The researcher was introduced to all Heads of Unit after which appointments were scheduled for center visits. On the scheduled day, the Heads of Unit were intimated about the purpose of the study and their voluntary participation was solicited. Following their interview, these Heads of Unit were asked to refer to other workers serving in the same work capacity to be interviewed on the same matter. Those identified were approached and verbal consent was also sought. All referred participants voluntarily agreed to take part in the study. Data collected from all participants were used in the final analysis as it was deemed appropriate since the population was small enough for total sampling to occur. Participants had formal original training in midwifery (in the case of the nurses) or were trained to offer midwifery services from schools of education (in the case of the community health practitioners).

\subsubsection{Data Collection Methods}

Data were collected using a semi-structured interview guide. Questions were as follows; (1) What do you think about fathers' involvement in Breastfeeding? Should fathers be involved in the breastfeeding process especially in helping women achieve 6 months exclusive breastfeeding (EBF)? (this question was asked to further clarify the first question). (2) How can fathers be involved in supporting 6 months of EBF? (3) What has been done to promote father involvement in this regard? (4) What needs to be done that has not already been done? (5) What may limit the successful implementation of father involvement in this regard? While these questions were used as a guide, participants were allowed to fully express themselves before being asked further questions.

\subsubsection{Trustworthiness}

The researcher earlier mentioned that this study was conducted as part of a larger mixed-method study underpinned by pragmatism. Preceding the study, the researcher discussed the feasibility of carrying out the study with experts in the field. During these discussions, issues such as the "nature of men" in the African context were discussed" which largely changed the focus of the study and the methods of data collection including instrumentation. Being a relatively new issue of interest within the study's society, a consensus was reached to adopt a more practical approach to inquiry to be able to obtain reasonable outcomes. Both researchers have worked on several 
community-based interventions in the study area and understood the stance of the wider examiners on the need for, first an in-depth look into the matter, in the development of a culturally friendly pilot intervention. The original idea was conceptualized by the first author based on a long-standing interest in breastfeeding and maternal-child health. Haven studied in that area, she understood that breastfeeding has a very strong social link. Communities vary in their daily modes of operation and as a social researcher, an open mind is required to penetrate communities. Hence the need arose to first conduct qualitative surveys to provide a broad basis for further research in this regard. Further on, trustworthiness was established and based on the following; credibility, transferability, dependability, confirmability as described by Korstjens and Moser (2018).

Credibility was established by giving feedback, following each interview. Responses were reiterated from the notes taken to confirm thoughts before moving on to the next interviewee. All interviews were held by the lead researcher in private rooms where participants were asked to "feel free" to express themselves based on their personal views.

Transferability was established by purposefully recruiting informed participants through an administrative focal person familiar with the nature of the health service in the area and leads the health operations in the area. Participants were selected based on the length of service of 5 years and above which ensured that they had, by the study time, sufficient experience and history in service to confidently and richly express themselves.

Confirmability has been established by providing a detailed methodology and rationale for data collection from the study participants. This detail provides information (as in the data collection sub-section) on how participants were identified and recruited, why they were recruited and how data was analysed to produce the findings herein.

\subsubsection{Ethical considerations}

Ethics committee approval was obtained from a Health Research Ethics Office within the study setting and permission was sought from the local government office. Voluntary participation was encouraged and anonymity and confidentiality were maintained by excluding names and location of participants from the audio recording.

\section{Data analysis}

Thematic analysis of data was carried out in a 6-step iterative process with steps overlapping (Braun \& Clarke, 2006; Maguire \& Delahunt, 2017). The first step was listening to the audio recordings and noting down the verbatim statements of each interviewee. This was followed by a review of transcripts for completeness of statements and notable gaps were filled as the audio recording was replayed. This was then followed by the development of themes produced from the interview guide questions. Further on, transcripts were reviewed to identify themes fitting with each code. Similar themes were regrouped within the related codes. Next, a general review of transcripts was carried out to ensure all themes had been captured. Data were cleaned and report produced to describe study findings.

\section{Findings}

The mean age and years of experience were 46 years and 18 years respectively. The health workers shared their perspectives about father involvement in the breastfeeding process including EBF support. The health workers shared views on father's involvement in breastfeeding support which included descriptions of father support, how father support could be encouraged, factors influencing father support, gaps in the health system hindering successful implementation of father-focused interventions and strategies for positive change.

Health worker's role in supporting fathers:

All participants acknowledged their unique role in the mechanism of enabling fathers to support mothers to breastfeed for a longer duration and up to 6 months and also noted that some efforts on their part have been ongoing. The following statements were made by the participants;

"It is the wife that will get home to explain to them and some will then say, "do you think it is possible ...won't the child drink water, since they did not hear it directly from the health worker's mouth, it will be difficult for them to comprehend... understand and even practice or give support to women. (Nurse, 12 years' experience -participant 4)

"During antenatal care, we do talk to them and some of them follow their wives. We talk to them about the importance of breastfeeding but it is not part of health care delivery" (Nurse, 20 years' experience-participant 7) Other findings were the recognition of breastfeeding challenges and advocates for the women. Participants also highlighted the resultant influence of the health worker. For instance, one of the participants noted that fathers are likely to better support if the route of information was through the health worker as disclosed by participant 4 .

"Yes, fathers should be involved, you know if you talk about breastfeeding it's a lot of stress on the part of the woman. So if the husband has an understanding of exclusive breastfeeding, there will be moral support, financial support...you know all support in all areas will be given to the woman. The man will understand that I am tired or have been asked to eat some kind of food and it will not go wrong on the side of the man and that is why we need to give men a detailed understanding of what it entails". 
Reflections of father support

Father's support was described in terms of social support. The most dominant support mentioned was that of offering instrumental support, not only in the form of finance and provision of food but also in terms of caring for the baby by carrying the baby when the mother is busy or helping to soothe a crying infant, attending post-natal care events such as immunization. In their own words, it was said,

"To me, they should not only give money, provisions, they should help in house chores when the mother wants to breastfeed the baby" (Community Health Extension Worker, 15 years' experience-participant 5)

"The husband will be able to provide enough food so the mother can breastfeed well....that is the major role of the husband. In the case of waking up in the night ...it is not that the father will say your baby is crying...oh(exclaims)..he should be able to carry the baby, pat the baby, put the baby on the chest as well..."(Nurse, 24 years' experience-participant 8 )

A participant emphasized the need for fathers to recognize the shared duty of caring between the man and woman and not to perceive that housework and other care activities are for women alone. Being present at antenatal was also highlighted. One of the health workers stated,

"When it's time for immunization, they should put it in mind, when the time comes they can say,... "I will take you there...I will bring you back home till the 6 months" (Nurse, 15 years' experience-participant 1)

Another form of support noted by participants was emotional support in the form of encouraging words and showing concern by calling home to determine the state of mother and baby while at work. These were disclosed by the community health extension worker and other participants.

Factors influencing father support

The participants were able to outline impeding factors to father support. The most dominant factors were the nature of work and lack of time. Others included a lack of knowledge about the need and importance of breastfeeding and EBF, cultural norms, lack of financial power, cosmetic reasons and influence of significant others, especially mothers/mothers in law. One of the participants while speaking implied that a lack of knowledge translates to a lack of practice. She stated that,

"So first and foremost, I will say ignorance...some of them do not know and when you do not know you do not practice". So they do not know about EBF. (Nurse, 12 years' experience -participant 4)

Another stated that "In the community that we find ourselves you find out that women are more committed in every area, financially to their family members. These men too, when they are not financially okay everything is destabilized. Majority when they don't have money and you ask for anything will not oblige. Their financial status gives them power" (Nurse, 15 years' experience-participant 1)

Health system gaps were also highlighted as key barriers to promoting father support, which include, a clear lack of integration into the current health care service delivery, lack of focused policy and lack of culture-friendly focused programmes and father focused interventions. As stated by two participants,

There is nothing in the hospital services...there is postnatal care...but this...maybe we call it father clinic...or paternal clinic... it has not been included in the services expected to be delivered by the health care institutions...I have not heard of any...It's not part of service delivery."(Nurse, 12 years' experience -participant 4)

".....Actually, there has never been....the only period has been at antenatal and post-natal when they want to enlighten them on family planning, that is when we get the men and that has also just been introduced..'(Community Health Extension Worker, 15 years' experience-participant 5)

Two of the participants underscored the influence of grandmothers and their beliefs and said, respectively;

" There are some they will say this baby does not breastfeed well give him food...give him water. Most nurses...doctors at home...you know those grandmas they will say in my own time my baby was taking this and that so there's nothing the woman can do...you know they will say for 6 months the baby will need water they think that the breast is so solid that the baby needs water"(Nursing, participant 8)

In our own culture if you are caring too much for your wife the mother will say ah! He has poisoned my child. That ah! How can you be doing this for your wife...so mothers...mothers in-law, some men will not be happy even if the mother is coming" (Nurse, 24 years' experience-participant 10)

Recommendations for promoting father support

The discussions prompted some suggestions for strategies to enable father involvement. The following were most frequently mentioned;

i) Utilizing the already existing antenatal services by purposefully inviting men during designated times to receive information on exclusive breastfeeding and also encouraging their presence during delivery to share in the experience to improve their knowledge and understanding and ultimately influence their practice of support. Follow-up visits could also be conducted to ascertain practice

ii) Integrating a "father-focused" health education element into health services delivery through policy acceptance and implementation of programmes beginning at the grassroots by utilizing already existing local festivals to communicate information using culturally friendly language.

iii) Collaborating with the media to communicate the importance of father support using all forms of media 
and social institutions.

iv) Finally, suggestions were given for the expansion of paternity leave nationwide to raise consciousness on the part of fathers on the need to actively engage in the breastfeeding process.

\section{Discussion}

In this study, health professionals did not only demonstrate enthusiasm towards encouraging father support in the breastfeeding process and but also acknowledged the additional role in facilitating active father support. Health professionals' acknowledgement of the father's role in the breastfeeding process has been noted as a key antecedent to promoting positive breastfeeding support and enhancing existing service delivery for breastfeeding mothers (Sheriff et al., 2014). Other research (Ledenfors \& Berterö, 2016) also confirms the relevance of health professionals in supporting fathers in matters of birth.

Descriptions of father support generally agreed with the wider literature on perceptions of father support Kansiime, Atwine, Nuwamanya \& Bagenda, 2017; Mithani, Premani, Kurji \& Rashid, 2015; Sheriff et al., 2014) albeit, two dominant elements of social support than what exists in the larger literature were noted in this study. To clarify the concept of "father support" to enhance promotional practice towards breastfeeding support, Sheriff et al. (2014) categorized father support into five essential domains namely, "Knowledge about breastfeeding, Positive attitude to breastfeeding, Involvement in the decision-making process, Practical support and Emotional support." (Sheriff et al., 2014) of which practical and emotional support, were the most highlighted domains in this study.

Several factors negatively influencing father support for breastfeeding have been discussed in the wider literature, and some were underscored in this study such as sociocultural factors in the form of the influence of other significant others outside of the mother-child-father triad, male dominance, absence of health system support, nature of work and myths, although breastfeeding myth was least emphasized.

A study in Pakistan reported similar findings. Sociocultural factors, lack of family support and lack of health service provision were sighted (Mithani et al., 2015). Although findings in this study closely matched findings in the Pakistani study, in aspects relating to the influence of significant others and father's economic status, findings slightly differed based on health service absence and poor attitude of the health practitioner. Health workers in this study indicated a lack of male-focused service but indicated personal efforts to involve fathers. Emphasis was made on the need for a health system inclusive framework to empower the health workers to more actively and engage fathers.

Overall, suggestions for moving forward father support in the study setting were in tandem with several other findings from previous research. Rollins et al. (2016) argued for a multi-streams supportive approach to promote breastfeeding. Participants in general, pointed to the need for more focused health education, the integration of a father support system into already existing health service delivery and the need to follow a bottom-up approach in health communication strategies. Rosin and Zakarija-Grković (2016) attempted to clarify the aim of the Babyfriendly initiative in developing integrated systems of breastfeeding support through the provision of services at the antenatal, perinatal and postnatal periods.

This study's findings are also in agreement with Brown and Davies (2014), who reported fathers' need for focused information to aid father support and also with Rosin and Zakarija-Grković (2016) who argued that policy consensus and political action are essential in the development of a framework for breastfeeding support. Policy support and political action were central themes in this study on paths to the successful promotion of father involvement.

The most dominant challenge as emphasized by the participants was the need for a support framework within the health system, therefore further research can investigate mechanisms of support for fathers of expectant and breastfeeding mothers at the policy level. Paternity leave was recently introduced in two states as a strategy to promote fathers' involvement in child care. Hence, future research is also needed to substantiate the effectiveness of the initiative, especially as it relates to breastfeeding support. The research was a pilot study conducted within one local government. Future research can be conducted across multiple sites to be able to improve the generalization of findings.

As a final note, the authors would like to note the following limitations. Only health workers who were readily available during data collection took part in the study. All participants were females therefore views from male health workers which could have provided a gender balanced perspective was absent. Future studies might consider closing the gender gap to assume a better balance of gender opinions. Although, it should be noted that the absence of male workers was not a deliberate act in this study. The study was done within the most natural setting and availability of the health workforce.

\section{Conclusion}

Health workers felt there is potential for the integration of father-focused breastfeeding programme into current service delivery. In order to actualize this, policy measures and other health system mechanisms of support will 
need to be standardized to foster the development and sustenance of father-focused breastfeeding initiatives. Currently, there is no provision for such services in the area. Hence, there is a need for raised awareness to make provisions for the actualization of father-friendly breastfeeding programmes.

\section{References}

1. Rollins, N.C. Bhandari, N., Hajeebhoy, N. Horton, S. Lutter, C.K. Martines, J.C. Piwoz, E.G. Richter, L.M. Victora, C.G., \& Lancet Breastfeeding Series Group. (2016). Why invest, and what it will take to improve breastfeeding practices? Lancet. 387(10017), 491-504

2. United Nations Children's Fund, (2019), Nutrition: breastfeeding. [online] Available: https://www.unicef.org/nutrition/index_24824.html

3. World Health Organization, (2019), Breastfeeding. [online] Available: https://www.who.int/nutrition/topics/exclusive_breastfeeding/en/

4. Johnson, A. M., Kirk, R., Rooks, A. J., \& Müzik, M. (2016). Enhancing breastfeeding through healthcare support: Results from a Focus Group Study of African American Mothers. Maternal and Child Health Journal, 20(Suppl 1), 92-102

5. National Bureau of Statistics. (2018). Report on the nutrition and health situation of Nigeria. [online] Available: https://www.unicef.org/nigeria/reports/national-nutrition-and-health-survey-nnhs-2018

6. National Bureau of Statistics (NBS) and United Nations Children's Fund (UNICEF). (2018). Nigeria: Multiple Indicator Cluster Survey 2016-17, National Survey Findings Report. Available: https://www.unicef.org/nigeria/reports/multiple-indicator-cluster-survey-2016-17-mics

7. Osibogun, O.O., Olufunlayo, T.F., \& Oyibo, S.O. (2018). Knowledge, attitude and support for exclusive breastfeeding among bankers in Mainland Local Government in Lagos State, Nigeria. International Breastfeeding Journal, 13(38), 1-7

8. Sholeye, O. O., Abosede, O. A., \& Salako, A. A. (2015). Exclusive Breastfeeding and its associated factors among mothers in Sagamu, Southwest Nigeria. Journal of Health Science, 5, 25-31

9. Agunbiade, O. M., \& Opeyemi, V. (2012). Constraints to exclusive breastfeeding practice among breastfeeding mothers in Southwest Nigeria: implications for scaling up. International Breastfeeding Journal, 7, $1-10$

10. Battersby, S. (2014). The role of the midwife in breastfeeding: Dichotomies and dissonance. British Journal of Midwifery, 22, 551-6

11. Nieuwoudt, S., \& Manderson, L. (2018). Frontline health workers and exclusive breastfeeding guidelines in an HIV endemic South African community: a qualitative exploration of policy translation. International Breastfeeding Journal, 13, 1-10

12. Brown, A., \& Davies, R. (2014). Fathers' experiences of supporting breastfeeding: Challenges for breastfeeding promotion and education. Maternal \& Child Nutrition, 10, 510-526

13. Rosin, S. I., \& Zakarija-Grković, I. (2016). Towards integrated care in breastfeeding support: a cross-sectional survey of practitioners' perspectives. International Breastfeeding Journal, 11, 1-17

14. Olaolorun, F., \& Lawoyin, T. (2006). Health workers' support for breastfeeding in Ibadan, Nigeria. Journal of Human Lactation, 22, 188-94

15. Utoo, B. T., Ochejele, S., Obulu, M. A \& Utoo, P. M. (2012). Breastfeeding Knowledge and Attitudes amongst Health Workers in a Health Care Facility in South-South Nigeria: the Need for Middle Level Health Manpower Development. Clinics in Mother and Child Health, 9(1), 1-5

16. Flood, J. L. (2017). Breastfeeding Supports and Services in Rural Hawaii: Perspectives of Community Healthcare Workers. Nursing Research and Practice, 2017(6041462), 1-14

17. Bhattacherjee, A. (2012) Social Science Research: Principles, Methods and Practices. South Florida, FL: USF Tampa Bay Open Access Textbooks Collection

18. Korstjens, I., \& Moser, A. (2018). Series: Practical guidance to qualitative research. Part 4: Trustworthiness and Publishing. European Journal of General Practice, 24(1), 120-124

19. Braun, V., \& Clarke, V. (2006). Using thematic analysis in psychology. Qualitative Research in Psychology, 3, 77-101

20. Maguire, M., \& Delahunt, B. (2017). Doing a Thematic Analysis: A Practical, Step-by-Step Guide for Learning and Teaching Scholars. All Ireland Journal of Higher Education, 9(3), 3351-33514

21. Sheriff, N., Panton, C., \& Hall, V. (2014). A new model of father support to promote breastfeeding. Community Practitioner, 87(5), 20-4

22. Ledenfors, A., \& Berterö, C. (2016). "First-time fathers' experiences of normal childbirth," Midwifery, 40, 26-31

23. Kansiime, N., Atwine, D., Nuwamanya, S., \& Bagenda, F. (2017). "Effect of Male Involvement on the Nutritional Status of Children Less Than 5 Years: A Cross-Sectional Study in a Rural Southwestern District of Uganda," Journal of Nutrition and Metabolism, 2017 (3427087), 1-9 
24. Mithani, Y., Premani, Z.S., Kurji, Z., \& Rashid, S. (2015). Exploring Fathers' Role in Breastfeeding Practices in the Urban and Semiurban Settings of Karachi, Pakistan. Journal of Perinatal Education, 24(4), 249-60 